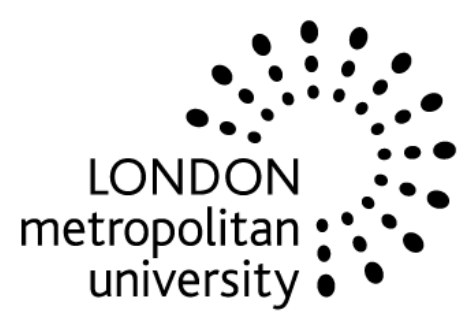

Centre for International Capital Markets

Discussion Papers

ISSN 1749-3412

Using Chebyshev Polynomials to Approximate Partial Differential Equations

Guglielmo Maria Caporale and Marrio Cerrato

No 2008-10 


\title{
USING CHEBYSHEV POLYNOMIALS TO APPROXIMATE PARTIAL DIFFERENTIAL EQUATIONS
}

\author{
Guglielmo Maria Caporale \\ Centre for Empirical Finance, Brunel University, London \\ Mario Cerrato \\ Department of Economics, University of Glasgow
}

April 2008

\begin{abstract}
This paper suggests a simple method based on a Chebyshev approximation at Chebyshev nodes to approximate partial differential equations. It consists in determining the value function by using a set of nodes and basis functions. We provide two examples: pricing a European option and determining the best policy for shutting down a machine. The suggested method is flexible, easy to programme and efficient. It is also applicable in other fields, providing efficient solutions to complex systems of partial differential equations.
\end{abstract}

JEL Classification: C63, G12

Keywords: European Options, Chebyshev Polynomial Approximation, Chebyshev Nodes

Corresponding author: Professor Guglielmo Maria Caporale, Centre for Empirical Finance, Brunel University, Uxbridge, Middlesex UB8 3PH, UK. Tel.: +44 (0)1895 266713. Fax: +44 (0)1895 269770.Email: Guglielmo-Maria.Caporale@brunel.ac.uk

Acknowledgements: We are grateful to Karim Abadir, Paresh Date, Brian Eales, and Geoff Rodgers for useful comments and suggestions. The usual disclaimer applies. 


\section{Introduction}

The study of partial differential equations (PDE) is a fundamental topic in applied mathematics, as well as in physics, natural science and finance. For example, in finance PDEs are used in arbitrage-based asset models. The widely cited Black and Scholes PDE, that must be satisfied by each European option within an arbitrage free market, is a canonical example. In this specific case, the PDE has an analytical solution; however, in many other interesting cases in finance, as well as in other fields, closed form solutions are very difficult to obtain and researchers rely on various numerical methods to obtain a solution. The study of these numerical methods represents the area of Computational Partial Differential Equations.

The most simple applicable algorithms to approximate PDEs rely on the concept of discretisation - that is, replacing the PDE of interest by a finite dimensional problem. However, replacing the PDE by a discrete model is not at all trivial and generally the choice of the finite dimensional model to be used depends on the properties of the mathematical model itself.

The development of high speed computers has made it easier to find accurate solutions to PDEs, even in the most extreme cases of very large systems of PDEs. In this study we show how to use polynomial methods to approximate PDEs. We shall only be focusing on second-order linear PDEs, although it would also be interesting to evaluate our methodology when dealing with non-linear types of PDEs. We leave this for future research.

Crack-Nicolson (CN) implicit schemes are amongst the most widely used approximation methods. However, their effectiveness is affected by the choice of time steps, which, very often, depends on the problem one is facing. Also, CN methods, in general, suffer from poor convergence.

The method suggested in this paper relies on polynomial interpolation to approximate the PDE characterising the option pricing problem. In our two applications, we use Monte Carlo methods to solve the boundary condition for the PDE, and fit the 
functional at Chebyshev nodes to estimate the coefficients ${ }^{1}$. The advantage of our approach is its flexibility, and the fact that it is easily implementable and since the functional, at least in the first empirical example, is approximated using deterministic nodes, the estimates of the coefficients are less scattered. In addition, our method is applicable in other fields, providing efficient solutions to complex systems of partial differential equations. These features make our approach very attractive. One reason why polynomial approximations of this type are underutilised (in comparison to direct ad hoc approximation methods) by applied researchers might be lack of familiarity. Therefore, in the following section, we provide some guidance on how to use them to solve systems of differential equations.

The layout of the paper is the following. Section 2 outlines the approximation method we advocate. Section 3 describes an option pricing valuation model. In Section 4 we apply our methodology to obtain the solution to the option pricing problem, as well as to an Investment Under Uncertainty problem. Section 5 summarises the main findings of this study and offers some concluding remarks.

\section{Polynomial Approximation}

In this section we describe in greater detail the approximation method adopted in this paper. Let $V \in \mathfrak{R}^{n+1}$ be a function defined on the interval $[a, b]$, which may well not be tractable analytically, and assume that $P$ is a polynomial that interpolates $V$ at the distinct $n+1$ points $s_{i} \in[a, b]$, with $P(s)=\sum_{i=0}^{n} c_{i} \phi_{i}(s)$. In order to solve the problem by approximation we need to define: (a) the family of basis functions to approximate the function $V$, (b) the interpolation nodes, $s_{i}$. In this section we show that Chebyshev polynomials in conjunction with Chebyshev nodes offer the best solution to our problem.

\footnotetext{
${ }^{1}$ Tzavalis and Wang (2003) use a similar approach based on Chebyshev approximation to approximate the optimal exercise boundary in the context of a stochastic volatility model. Their method also relies on extrapolation procedures.
} 
Theorem 1: if $V \in \mathfrak{R}[a, b]$, then for all $\varepsilon>0$ there exists a polynomial $P(s)$ such that $\forall s \in[a, b]|V(s)-P(s)| \leq \varepsilon$.

Remark 1. The above theorem is known as the Weierstrass theorem. It states that any continuous function can be approximated with a certain degree of accuracy by using a polynomial. Although very important theoretically, this theorem is of little practical use since it does not give any indication of what polynomial is the most appropriate to use, or even what order polynomial is needed to achieve a certain degree of accuracy.

The error made by using a polynomial of order $n$ to approximate the function given in Theorem 1 can be easily calculated as:

$$
V(s)-P(s)=\frac{1}{n+1} V^{(n+1)}(\varepsilon) \prod_{i=0}^{n}\left(s-s_{i}\right)
$$

The objective of using such an efficient polynomial consists in choosing a set of nodes $s_{i}$ so as to make the term $\prod_{i=0}^{n}\left(s-s_{i}\right)$ as small as possible (Judd, 1998). One possibility is to approximate the function $V$ at the n-evenly spaced nodes. However, it is well known that in general, even for smooth functions, polynomials of this type do not produce very good approximations. ${ }^{2}$ Therefore, we suggest approximating the function over the interval $[a, b]$, at the Chebyshev nodes defined as:

$$
s_{i}=\cos \left(\frac{2 i+1}{2 n+2} \pi\right), i=0,1, \ldots, n
$$

Our approach can be justified by appealing to Rivlin's theorem, stating that Chebyshev node polynomial interpolants are nearly optimal polynomial approximants (Rivlin, 1990),

\footnotetext{
${ }^{2}$ A classic example is Runge`s function (Rivlin, 1990).
} 
and has been shown to perform well empirically (Rivlin, 1990). Chebyshev nodes are also known to possess a further convenient property, i.e. equi-oscillation ${ }^{3}$ (Judd, 1998).

As important as the choice of the nodes interpolants is that of a family of functions from which the approximant $P$ will be drawn. We suggest using a Chebyshev polynomial. This is defined as ${ }^{4}$ :

$$
\Gamma_{i}(s)=\cos (i a \cos (s)) \quad i=0,1, \ldots, n
$$

with $\Gamma_{0}(s)=1, \Gamma_{1}(s)=s$, and $\Gamma_{n+1}(s)=2 s \Gamma_{n}-\Gamma_{n-1}(s)$

Therefore:

$$
V(s)=\sum_{i=0}^{n} c_{i} \Gamma_{i}(s)
$$

$$
\text { where } c_{0}=\frac{1}{n+1} \sum_{i=0}^{n} V\left(s_{k}\right) \text { and } c_{i}=\frac{2}{n+1} \sum_{i=0}^{n} V\left(s_{k}\right) \cos \left(i a \cos \left(s_{k}\right)\right), i=1, \ldots, n
$$

A Chebyshev basis polynomial, in conjunction with Chebyshev interpolation nodes, produces an efficient interpolation equation which is very accurate and stable over $n$. However, in our case, to solve the problem given by (1), the polynomial we choose should be able to replicate, not just the function $V$ at $s_{1}, s_{2}, \ldots, s_{n}$, but also its derivatives $s_{1}^{\prime}, s_{2}^{\prime}, \ldots, s_{n}^{\prime}$. Therefore the approximant that solves our problem can be defined as follows ${ }^{5}$ :

$$
\begin{aligned}
& \sum_{i=1}^{n} c_{i} \Gamma_{i}(s)=V\left(s_{i}\right), \forall_{i}=1, \ldots, n_{1} \\
& \sum_{i=1}^{n} c_{i} \Gamma_{i}^{\prime}\left(s^{\prime}\right)=V^{\prime}\left(s_{i}\right), \forall_{i}=1, \ldots, n_{2}
\end{aligned}
$$

\footnotetext{
${ }^{3}$ This property states that the maximum error of a cubic function, for example, shall be reached at least five times, and the sign of this error should alternate between the interpolation points.

${ }^{4}$ Note that in this application we use the general formula for the Chebyshev basis,; however, there exists also a recursive formula.

${ }^{5}$ Note that, although one can also use Hermite polynomials to approximate the functional and the slopes, the latter are inefficient (Judd, 1998).
} 
with $n_{1}+n_{2}=n$.

Once the basis functions (approximants) have been chosen and the approximant nodes defined, the basis coefficients $c_{i}$ can be obtained. If we define the following ChebyshevVandermode type matrix $\mathrm{T}$ :

$$
\mathrm{T}=\left[\begin{array}{cccc}
\Gamma_{0}\left(s_{1}\right) & \Gamma_{1}\left(s_{1}\right) & \cdot & \ldots \Gamma_{n-1}\left(s_{1}\right) \\
\Gamma_{0}\left(s_{2}\right) & \Gamma_{1}\left(s_{2}\right) & \cdot & \Gamma_{n-1}\left(s_{2}\right) \\
\cdot & \cdot & \cdot & \cdot \\
\Gamma_{0}\left(s_{n}\right) & \Gamma_{1}\left(s_{n}\right) & \cdot & \Gamma_{n-1}\left(s_{n)}\right.
\end{array}\right]
$$

then the coefficients $c_{i} ; c=\left(c_{0}, c_{1}, \ldots, c_{n-1}\right)^{\prime}$ of $V(s)$ solve Tc$=V$, with $\Gamma_{i j}=\Gamma_{i}\left(s_{i}\right)$ being the $\mathrm{j}$ basis function evaluated at the $\mathrm{i}$-th interpolation node. When $s$ is allowed to vary over some other interval, say $[t, T] \neq[-1,1]$, we rescale the value of $s$ to $s^{*}$ where $s^{*}=\frac{1}{2}((T-t) s+(T+t))^{6}$.

As an example of using different basis functions, we anticipate some of the empirical results presented in the next section and after pricing an European option we calculate the approximation error. We use two different basis functions (i.e. Chebyshev basis and spline basis). The approximation error is shown in Figures (1-2).

\section{Insert Figures (1-2) here}

As can be seen, when the approximation is calculated using Chebyshev basis functions the error is of the order of $1 \times 10^{-15}$ for a polynomial of order 20 . Spline functions do not

\footnotetext{
${ }^{6}$ An interesting issue here is the non-singularity of the Vandermode matrix over Chebyshev basis as above. In theory, there is no guarantee that the matrix is non-singular. However, in practice, in general applications such as ours, we can conjecture that as long as the number of indeterminates exceeds the sparsity with respect to $\mathrm{T}$, non-singularity should hold. Alternatively, we suggest two ways to overcome the problem: (a) simply use the singular value decomposition of $\mathrm{T}$; (b) use the generalised Vandermode matrix over Chebyshev. In fact, for this type of matrix Werther (1993) proves that, as long as the indeterminates take a value $[1, \infty]$, the generalised Vandermode matrix over Chebyshev basis is non-singular.
} 
achieve a comparable degree of accuracy even increasing the order of the polynomial to 30. Furthermore, Chebyshev polynomials exhibit their usual oscillation which appears fairly evenly over the interval we have considered. On the other hand, spline polynomials exhibit larger oscillations at the edges of the interval.

\section{The Valuation Model}

This section outlines an option pricing problem to which our method will be applied. Suppose that the price of a non-dividend-paying asset in period 0 is $S_{0}$, and denote with $K$ the strike price of a put option written on that asset.

Assumption 1: We assume that the option value depends on the stock price at expiry of the option and time, $V_{t}\left(S_{t}, t\right)$.

Suppose also that the process for $S$ is described by the following geometric Brownian motion:

$$
d S_{t}=S_{t} r d t+S_{t} \sigma d Z_{t}
$$

where $d Z$ is a standard increment of a Wiener process, and $\sigma$ the variance parameter.

We can expand $E(d V) \frac{1}{d t}$, using Ito`s Lemma and the stochastic process above to obtain:

$$
r V=r S V_{S}+V_{t}+\frac{1}{2} S^{2} \sigma^{2} V_{S S}
$$

where $V_{(.)}$represents the derivative with respect to the argument in the subscript.

All European options, in absence of arbitrage, must satisfy Equation (3). A call option will have at expiry a payoff given by $S-K$, if $S>K$, while for a put option at expiry the payoff is $K-S$, if $K>S$. Therefore, in our specific case, the boudary condition is given by 


$$
V(S, t)=\max (0, K-S)
$$

If we set the value function above $V(s, t) \approx \phi(s) c(t)$, where $\phi$ is a suitable basis for an $\mathrm{n}$ dimensional family of approximating functions and $c(t)$ is an n-vector of time-varying coefficients, equation (3) can be re-written as follows:

$$
\phi(s) c^{\prime}(t) \approx\left[r s \phi^{\prime}(s)+\frac{1}{2} \delta^{2} s^{2} \phi^{\prime \prime}(s)-r \phi(s)\right] c(t) \approx \psi(s) c(t)
$$

To determine $c(t)$, we select n-values (nodes) of $s, s_{i}$, and solve (4) for that particular set of values. Given the n-dimensional family of basis functions chosen, (5) can now be re-written in the form of a system as follows:

$$
\Phi c^{\prime}(t)=\Psi c(t)
$$

where $\Phi$ and $\Psi$ are two $n \times n$ matrices.

Once the coefficients have been obtained as in (6), to price the financial option, first we use the process in (2) to obtain estimates of (4). Then, we multiply it by the estimated coefficients. Averaging gives the price of the option.

\section{Two Empirical Examples: Option Pricing and Investment Under Uncertainty}

In order to show how to use in practice the proposed method we now provide two examples. The first is the valuation problem described in section 2. We use the proposed methodology to value an European put option written on a stock. In this case, we can compare our empirical results with the Black and Scholes closed-form solution. We use the absolute error (ASE) as a measure of accuracy.

Table 1 shows the results for the entire set of options considered. We also report the results using the Black and Scholes method (1973 - B\&S henceforth). 
Insert Table 1 here

We fit (4) using the first twenty Chebyshev basis to estimate the parameters $c_{i}$ in (4) The basis number has been chosen using Theorem 6.4.2 in Judd (1998). Once the coefficients have been estimated, we estimate the boundary condition in (3) by simulating 200,000 paths for the stock. We can see that our method produces rather accurate option prices. This is confirmed by the absolute error reported at the bottom of Table 1 .

The second empirical example we consider is taken from the theory of Investment Under Uncertainty. The problem is to decide when it is optimal to shutdown a machine, assuming that there is no maintenance cost for maintaining it alive. Suppose that $\pi(t)$ is the profit generated by the machine at time $t$, and suppose that it follows the Brownian motion process below

$$
d \pi(t)=a d t+\sigma d z, \quad \pi(0)=\pi_{0}
$$

where $a$ is the rate of depreciation of the investment, $\sigma$ is the volatility of profit, and $d z$ is an increment of a Wiener process.

We report three paths of the process in Figure 3. We consider the parameters reported at the bottom of Table 3, and the time $T=10$ has been divided in one-hundred time steps. It appears that the investment will, in general, produce a loss before 3 years. However, the decision to shutdown the machine cannot be only taken by looking at these dynamic paths. In fact, this is a more complex problem since once the machine has been shutdown it cannot be re-started again, that is, the investment is irreversible. Therefore, one has to consider the optimal policy to decide when it is convenient to shut it down.

Following Dixit and Pindyck (1994), suppose that $F(\pi, t)$ is a claim on the profit flow, $\pi$, and suppose it is determined as 


$$
F(\pi, t)=\max _{\tau \in \Gamma} E \int_{0}^{T} e^{-r t} \pi(t) d t
$$

where $r$ is the risk free rate of interest, $T$ is the time and $\tau \in \Gamma$ is a random stopping time.

Suppose that the machine can be shut down only up to time $\bar{T}$. After that time it has to run forever. We assume that if it is shut down it cannot be re-installed, which highlights the irreversibility of the investment.

The Bellman Equation for the optimal stopping problem can be written as

$$
F(\pi, t)=\max \left(0, \pi d t+(1-r d t)^{-1} E[F(\pi+d \pi, t+d t)]\right.
$$

Therefore, if the machine is shut down the profit will be zero. If it is kept alive, then profit is given by the conditional expectation in (9). One can show that, for this case, the value function $F$, in the continuation region, satisfies the following Bellman equation

$$
r F=\pi+a F_{\pi}+1 / 2 \sigma^{2} F_{\pi \pi}
$$

where $F_{(.)}$is the derivative with respect to the sub-script.

Under the assumption that, if not abandoned by $\bar{T}$, the machine has to run forever, the terminal condition can be written as $F(\pi,-\bar{T})=\max \left(0, a / r^{\wedge} 2+r / \pi\right)$.

\footnotetext{
7 Note that we estimate these coefficients using Chebyshev nodes and Chebyshev basis.
} 
We can still use the same approach as in Section (2) to solve this optimal stopping problem. However, now solving (10) at each stopping times require more effort. One possibility would be to use finite difference methods. However, this approach turns out to be very time consuming. On the other hand, using Richardson extrapolation methods would increase the speed but at a cost of, sometimes, poor convergence. The method described in Section (2) can be adapted to this specific case. In fact, it is very similar in spirit to the Least-Squares algorithm introduced in Longstaff and Schwartz (2001). The coefficient $c$ in Section 2 are still estimated using Chebyshev polynomials and the same approach as before but now the interpolant nodes are projected using $(7)^{8}$. We assume that there is a threshold at $\$ 0.87$ and once it has been reached one has to decide if it is convenient to shut down the machine. Using 200 time steps, four Chebyshev basis functions and one hundred thousands replications, we estimate that it is optimal to shut down the machine soon after its second year of life. This empirical result is in line with the three paths shown in Figure (3).

\section{Conclusions}

In this study we suggest a simple method to approximate partial differential equations based on a Chebyshev approximation at Chebyshev nodes. We provide two empirical examples, in both cases obtaining highly satisfactory results. The first example consisted in pricing an European put option, the second in solving an optimal stopping problem. In the latter case stochastic rather than determinist nodes were used to approximate the functional. Our method is simple to apply and to extend and provides a reliable framework which can be used either for pricing more complex derivative instruments or in many interesting cases in economics and in other fields. Our future research will carry out further applications to provide additional evidence of the advantages of our approach.

\footnotetext{
${ }^{8}$ We have also tried to estimates the parameters using Chebyshev nodes but in this specifi case we obtained a poor fit. Therefore we suggest, when dealing with optimal stopping problems, not to use Chebyshev nodes.
} 


\section{References}

Abadir, K., and M., Rockinger, 2003, "Density Functionals, With An Option-Pricing Application", Econometric Theory, 4.

Black, F. and M. Scholes, 1973, "The Pricing of Options and Corporate Liabilities", Journal of Political Economy, 81, 637-654.

Chang, C., C.S. Chung and R.C. Stapleton, 2002, "Richardson Extrapolation Techniques for the Pricing of American-Style Options", Department of Finance, The Management School, National Central University, Taiwan, Working Paper No. 3.

Dixit, A., 1992, "Investment and Hysteresis", Journal of Economic Perspectives, $6,107-132$

Dixit, A., and S. Pindyck, 1994, "Investment Under Uncertainty", Princeton University Press.

Judd, K.,1998, "Numerical Methods in Economics", Massachusetts Institute of Technology Press.

Longstaff, F.A. and E.S. Schwartz, 2001, "Valuing American Options by Simulation: A Simple Least-Squares Approach", Review of Financial Studies, 14, 1, 113 147.

Pindyck, R.S., 1991, "Irreversibility and the Explanation of the Investment Behaviour", in D. Lund and B., Oksendal (eds.), Stochastic Models and Option Values,, Amsterdam, North Holland.

Rivlin, Theodore J., 1990, "Chebyshev Polynomials: From Approximation Theory to Algebra and Number Theory", $2^{\text {nd }}$ edition, New York: John Wiley and Sons.

Tzavalis, E., and S. Wang, 2003, "Pricing American Options Under Stochastic Volatility: A New Method Using Chebyshev Polinomials to Approximate the Early Exercise Boundary", Working Paper No. 488, Queen Mary, University of London, Department of Economics.

Werther, T.,1993,“Generalized Vandermode Determinants over the Chebyshev Basis”, International Computer Science Institute, 1947, Center Street, Berkeley, California. 


\section{Tables and Figures}

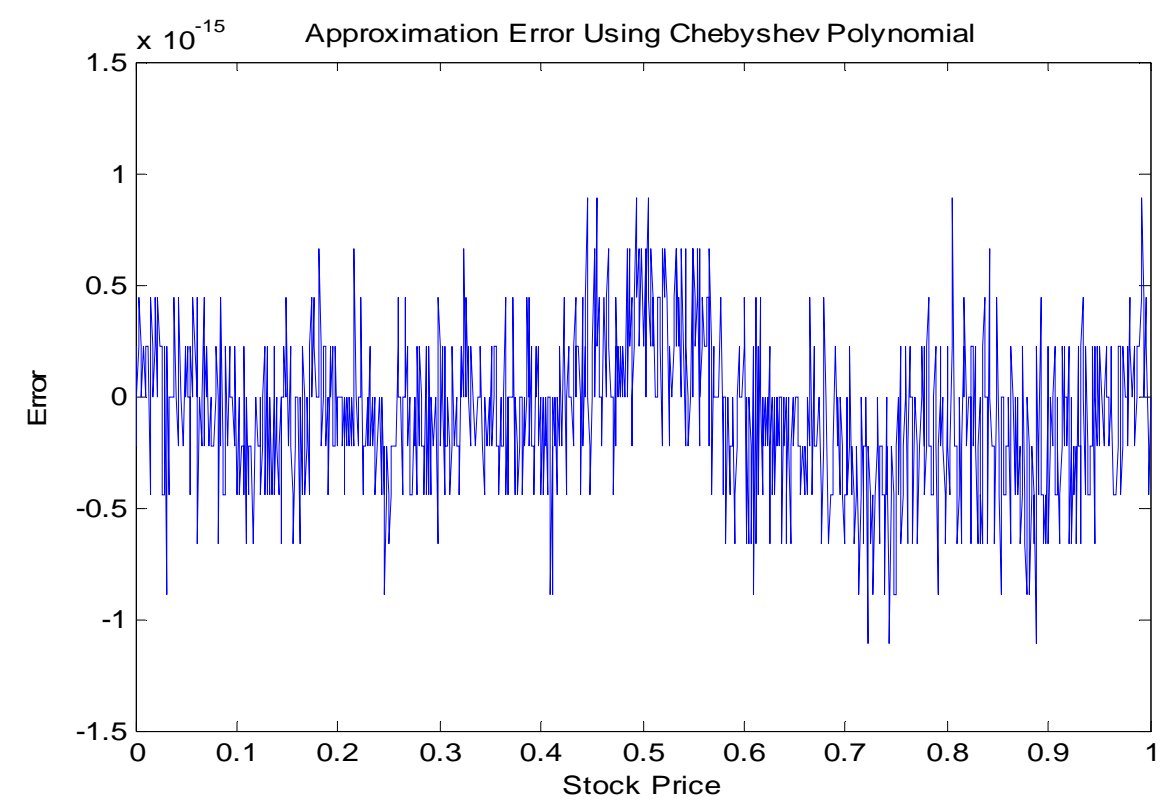

Figure 1

Approximation error using (8) and Chebyshev polynomial when volatility is equal to 0.2 and the interest rate is 0.048 .

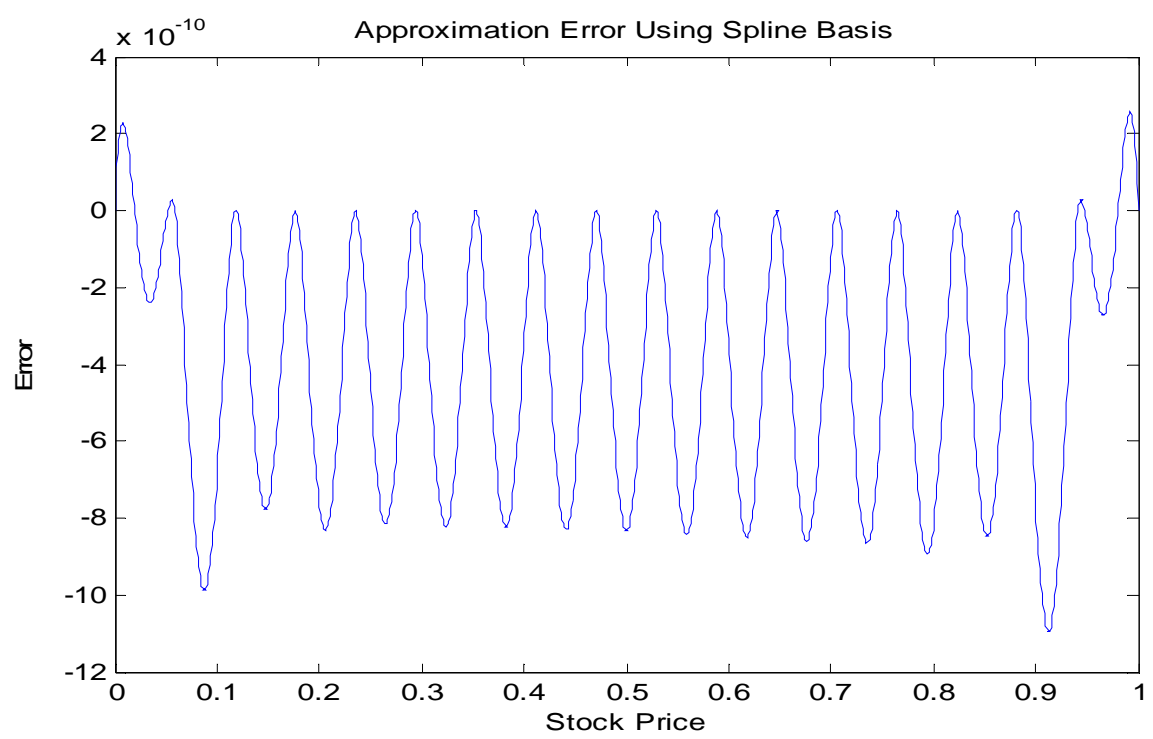

Figure 2

Approximation error using (8) and splines basis when volatility is equal to 0.2 and the interest rate is 0.048 


\begin{tabular}{|ccccc|}
\hline $\mathbf{K}$ & Sigma & $\mathbf{t}$ (yrs) & $\mathbf{B} \& \mathbf{S}$ & $\mathbf{C C}(\mathbf{2 0 0 8})$ \\
40 & 0.2 & 0.0833 & 0.8403 & 0.838 \\
40 & 0.2 & 0.3333 & 1.5221 & 1.472 \\
40 & 0.2 & 0.5833 & 1.8812 & 1.802 \\
45 & 0.2 & 0.0833 & 4.8399 & 4.837 \\
45 & 0.2 & 0.3333 & 4.7804 & 4.771 \\
45 & 0.2 & 0.5833 & 4.84 & 4.813 \\
40 & 0.3 & 0.0833 & 1.2988 & 1.297 \\
40 & 0.3 & 0.3333 & 2.4275 & 2.426 \\
40 & 0.3 & 0.5833 & 3.0634 & 3.062 \\
45 & 0.3 & 0.0833 & 4.9796 & 4.976 \\
45 & 0.3 & 0.3333 & 5.5288 & 5.517 \\
45 & 0.3 & 0.5833 & 5.9723 & 5.946 \\
40 & 0.4 & 0.0833 & 1.7575 & 1.758 \\
40 & 0.4 & 0.3333 & 3.3336 & 3.3335 \\
40 & 0.4 & 0.5833 & 4.2473 & 4.246 \\
45 & 0.4 & 0.0833 & 5.236 & 5.235 \\
45 & 0.4 & 0.3333 & 6.3767 & 6.359 \\
45 & 0.4 & 0.5833 & 7.1654 & 7.131 \\
$\mathrm{ABE}$ & & & & 0.0151 \\
\hline
\end{tabular}

Table 1

Column 4 shows the results using the Black and Scholes (1973) method.

Column 5 shows the results using our suggested method (Caporale and Cerrato - CC).

RMSE at the bottom of the table is the root mean square error. 


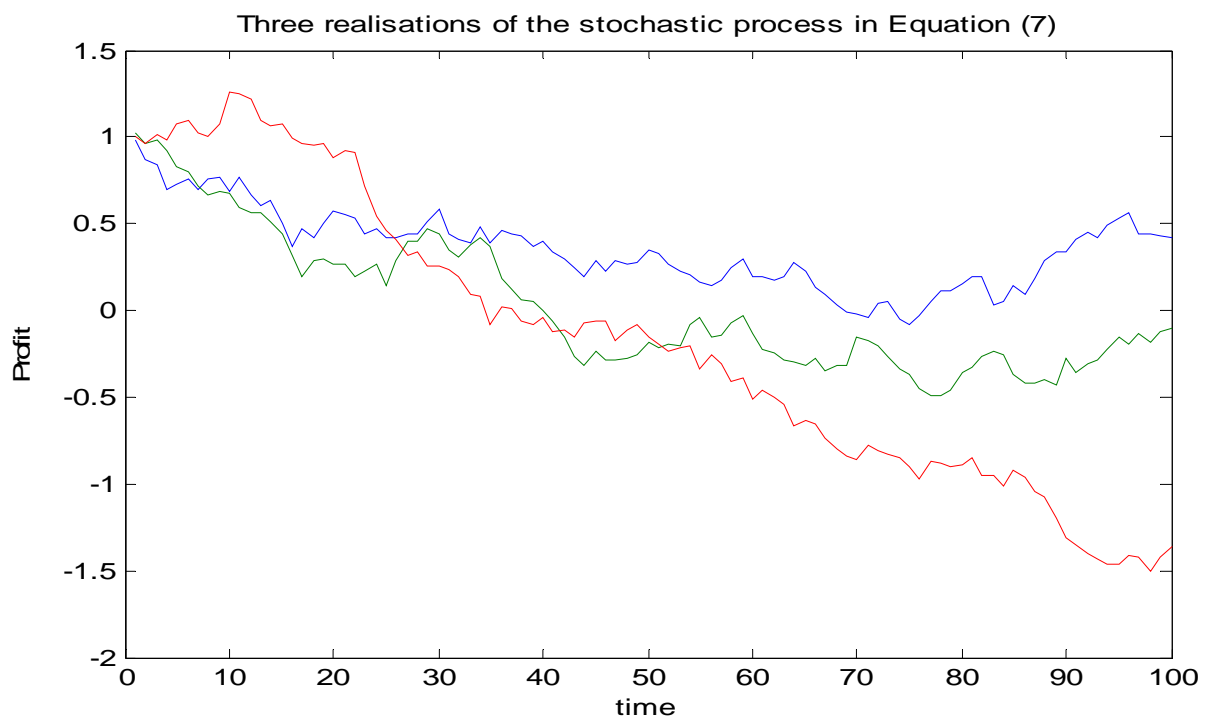

Figure 3

The stochastic process in Figure 3 has been simulated using $a=-0.1, \sigma=0.2$, and $\pi_{0}=1$. 